\title{
Clinical assessment of intraocular pressure: a whole-eye dynamic model
}

Francesca Stefanoni ${ }^{1}$, Alon Harris ${ }^{3}$, Marcela Szopos ${ }^{2}$, Christophe Prud'homme², Riccardo Sacco ${ }^{1}$, Dario Messenio ${ }^{4}$, Maria Laura Costantino ${ }^{1}$, Giovanna Guidoboni ${ }^{2}$

${ }^{1}$ Department of Mathematics, Politecnico di Milano (Polythecnic University of Milan), Milan, Italy; 'Institute for Advanced Mathematical Research, UMR 7501, National Center for Scientific Research, University of Strasbourg, Strasbourg, France; ${ }^{3}$ Eugene and Marilyn Glick Eye Institute, Indiana University School of Medicine, Indianapolis, IN, USA; ${ }^{4}$ Eye Clinic, Department of Clinical Science - ASST Fatebenefratelli Sacco, University of Milan, Milan, Italy

\section{Abstract}

The aim of this work is to provide a quantitative description of how geometrical and material properties of ocular tissues and fluids influence intraocular pressure (IOP).

Keywords: intraocular pressure (IOP), glaucoma management, mathematical modeling, sensitivity analysis

\section{Introduction}

IOP assessment is a simple, quick and non-invasive test that can be performed in any ophthalmology clinic. IOP alterations are associated with many diseases, such as glaucoma, and it is therefore of great clinical interest to identify the factors influencing IOP levels. Indeed, it is well known that geometrical and material properties characterizing ocular tissues and fluids influence IOP. In this context, the main goal of this work is to address the need for quantitative understanding of the influence of patient-specific geometrical and material properties of ocular tissues and fluids on IOP by means of mathematical modeling.

Correspondence: Francesca Stefanoni, Department of Mathematics, Politecnico di Milano, via Bonardi 9, Milano, Edificio 14 "La Nave" Campus Leonardo del Politecnico di Milano, Milan, Italy.

E-mail: Francesca.stefanoni91@gmail.com 


\section{Methods and results}

The model aims to be an electrical analog of the physiology of the eye, including various details regarding structural mechanics and fluid dynamics. Interestingly, pressure values inside the eye exhibit a static component, mainly due to the balance of aqueous humor ( $\mathrm{AH}$ ) inflow and outflow, and a dynamic component, mainly due to blood flow oscillations. We considered both static and dynamic components, and in particular, we investigated and quantified the influence of tissue (vitreous humor, cornea, and sclera) deformability and blood flow pulsatility on the dynamic measurements of IOP. Model calibration and validation have been performed using published data. The model has been implemented using the open access software OpenModelica (Open Source Modelica Consortium: Linköping, Sweden) in order to facilitate further model extensions and connections with other models already available for different parts of the eye.

\subsection{Basic modeling assumptions}

We defined the total volume of the eye as the sum of volumes of aqueous humor (anterior chamber), choroidal blood, and internal structures (vitreous humor, lens, and iris):

$$
V_{\text {tot }}=V_{A H}+V_{\text {blood }}+V_{\text {struc }}
$$

In our model, the analog of volume is the electrical charge, whereas the analog of volumetric flow rate is the electrical current. In the following sections, we review three models that simulate IOP under different assumptions. The models can be thought of as successive steps towards the modeling of whole-eye dynamics.

\subsection{Static conditions}

As a first step, pressure is assumed to be uniform inside the eye, while choroid and structure volumes are assumed to be given constants. The steady-state value of IOP is computed as the solution of the equaticon that describes the balance between $\mathrm{AH}$ production and drainage, as in Szopos et al.: ${ }^{1}$

$$
\frac{d V_{A H}}{d t}=J_{\text {in }}-J_{\text {out }}=0
$$

$\mathrm{AH}$ inflow is due to the processes of ultrafiltration and ionic secretion, whereas the outflow occurs through the trabecular and uveoscleral pathways. The balance leads to a scalar third order polynomial equation in the sole unknown IOP:

$$
L\left[(c B P-I O P)-\sigma_{p} \Delta \pi_{p}-\sigma_{s} \Delta \pi_{s}\right]=\frac{1}{R_{0}[1+Q(I O P-E V P)]}(I O P-E V P)+\frac{k_{1}}{k_{2}+I O P} I O P
$$

Using published data for the parameters (see Table 1), the value for the steady-state 
Table 1. Control state values for the parameters

\begin{tabular}{|l|l|l|}
\hline Parameter & Value and Unit & Meaning \\
\hline$L$ & $0.3 \mu \mathrm{l} / \mathrm{min} / \mathrm{mmHg}$ & hydraulic conductance \\
\hline$C B P$ & $27.5 \mathrm{mmHg}$ & ciliary blood pressure \\
\hline$\Delta \pi_{p}$ & $25 \mathrm{mmHg}$ & oncotic pressure difference \\
\hline$\sigma_{p}$ & $1[-]$ & protein reflection coefficient \\
\hline$\Delta \pi_{s}$ & $-450 \mathrm{mmHg}$ & osmotic pressure difference \\
\hline$\sigma_{s}$ & $0.0515[-]$ & low-molecular component coefficient \\
\hline$E V P$ & $8 \mathrm{mmHg}$ & episcleral vein pressure \\
\hline$R_{o}$ & $2.2 \mathrm{mmHg} \mathrm{min} / \mu \mathrm{l}$ & resistance when pressure gradient equals zero \\
\hline$Q$ & $0.012 \mathrm{mmHg}$ & outflow obstruction coefficient \\
\hline$k_{1}$ & $0.4 \mu \mathrm{l} / \mathrm{min}$ & maximum uveosclerar flow rate \\
\hline$k_{2}$ & $5 \mathrm{mmHg}$ & pressure corresponding to half max uveoscleral flow rate \\
\hline
\end{tabular}

Baseline values for the parameters: typical conditions of a healthy eye.

IOP found by OpenModelica is $14.9527 \mathrm{~V}$, which is perfectly in the range of physiological IOP measured in the clinics. We performed a deterministic sensitivity analysis on Equation (3) showing that the major influence on IOP is given by $\Delta \pi_{p}, \Delta \pi_{s}$, and $\mathrm{cBP}$, as shown in Figure 1.

\subsection{Dynamic conditions}

\subsubsection{Ocular hemodynamics}

We introduced a non-stationary component in the model starting from the model presented in Kiel et al. ${ }^{2}$ The new assumption with respect to the previous model is that IOP depends on $V_{\text {tot }}$ as in Equation (4), the given functions of time, $V_{c h}(t)$, and its first derivative, $G_{c h}(t)$, estimated as in Krakau. ${ }^{3}$ The new model is thus described by the following system of equations:

$$
\left\{\begin{array}{c}
\frac{d V_{\text {tot }}}{d t}=J_{\text {in }}-J_{\text {out }}+G_{c h} \\
\text { IOP }=\overline{\mathrm{IOP}} 10^{\mathrm{V}(\mathrm{Vtot}-\mathrm{Vtot})}
\end{array}\right.
$$

where $\gamma$ is a constant equal to $0.0215 \mu^{-1}$ and overlined variables indicate baseline values according to literature $\left(15 \mathrm{mmHg}\right.$ and $7 \mathrm{ml}$, respectively, for $\overline{I O P}$ and $\left.\overline{V_{\text {tot }}}\right)$. Changes in choroidal volume due to blood flow variations, namely $G_{c h}(t)$, are given by a sinusoidal current source. Changes in total volume, namely $d V_{\text {tot }} / d t$, are modeled by a capacitor, accounting for energy storage due to structural deforma- 


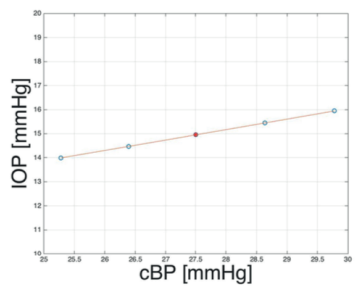

(a) Variations in $c B P$

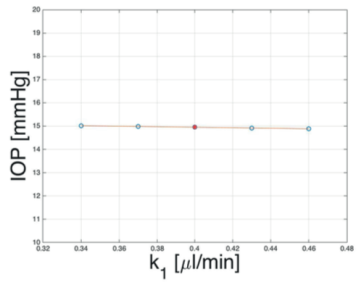

(d) Variations in $k_{1}$

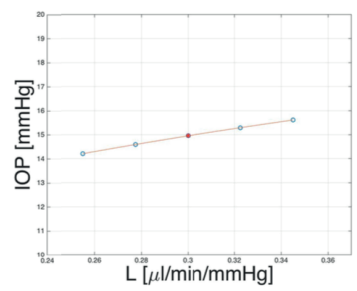

(b) Variations in $L$

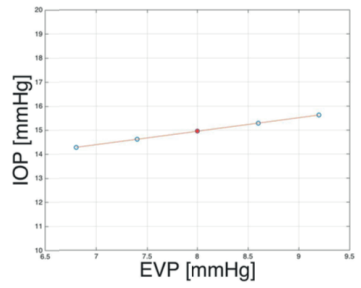

(e) Variations in EVP

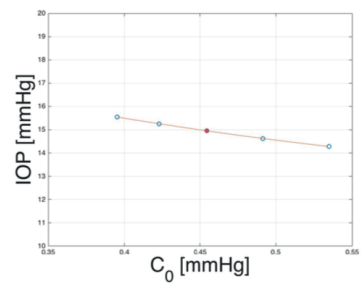

(c) Variations in $C_{0}$

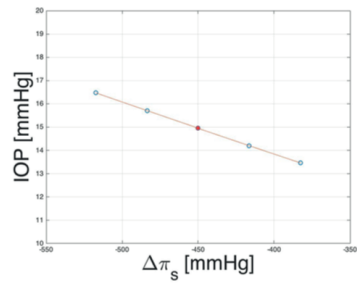

(f) Variations in $\Delta \pi_{s}$

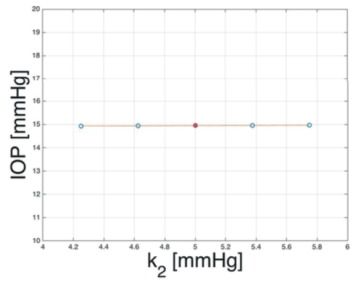

(g) Variations in $k_{2}$

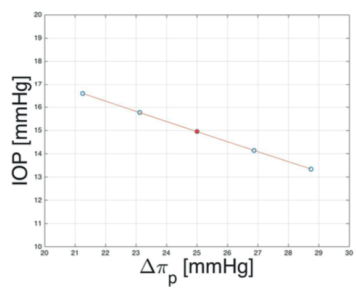

(h) Variations in $\Delta \pi_{p}$

Fig. 1. Influence of different factors on IOP. Red dots indicate control state values for the variables and IOP; blue dots indicate samples used in the circuit.

bility of the eye. For the calibration of the current generator, we chose a frequency equal to the one of the clinically-measurable IOP wave, namely $1.1 \overline{3} S^{-1}$. Oscillations in IOP indeed reflect changes in eye volume due to changes in the intraocular blood volume induced by the arterial blood pulse; thus, this frequency is that of the cardiac cycle. The amplitude value has been chosen to be $10.3166 \mu \mathrm{SS}^{-1}$, as in Krakau. ${ }^{3}$

\subsubsection{Ocular deformability}

As a first step, we estimated a constant value for the capacity by means of a linearization of the relationship between $I O P$ and $V_{\text {tot }}$ shown in Equation (4):

$$
C=\frac{\Delta V_{\text {tot }}}{\Delta I O P}=\frac{1}{\gamma \overline{I \overline{O P}}}=1.35 \mu / \mathrm{mmHg}^{-1}
$$

Note that $\gamma$ in Equation (5) is the same as in Equation (4), but with the relation expressed in natural logarithm. $\gamma$ accounts for the elastic proprieties of the cornea, 


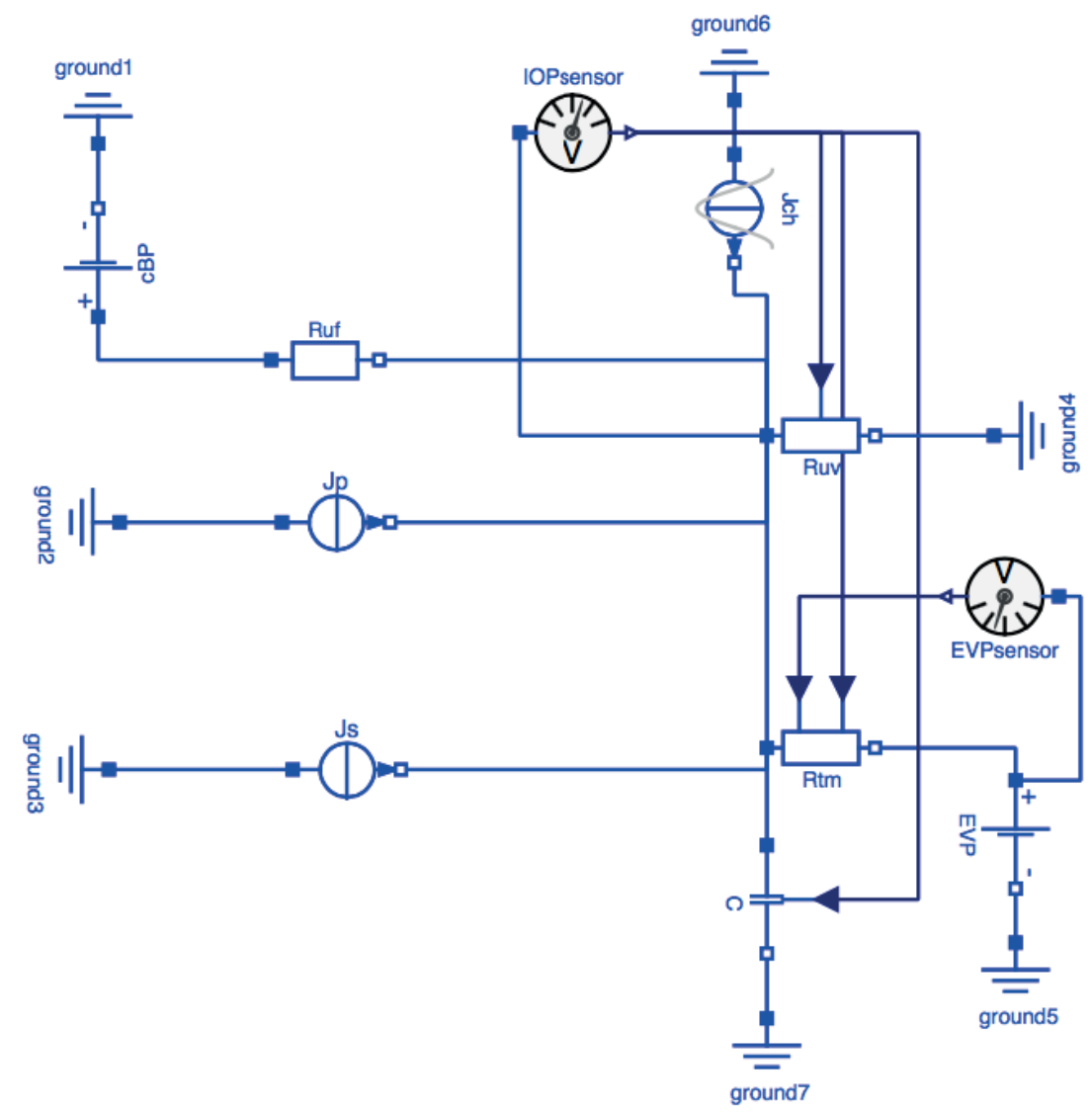

Fig. 2. OpenModelica electrical circuit for the dynamic component of IOP.

sclera, and other boundary structures of the eye, and is named ocular rigidity as in Friedenwald. ${ }^{4}$

Then we estimated a more precise non-linear value of the capacity as a function of IOP:

$$
C=\frac{1 / v \log \left(\frac{\mathrm{IOP}}{\overline{\mathrm{IOP}}}\right)}{\mathrm{IOP}-\overline{\mathrm{IOP}}}
$$

The circuit implemented in OpenModelica and the resultant IOP wave are shown in Figures 2 and 3, respectively. The sinusoidal IOP has an average value of 15.0179 $\mathrm{mmHg}$ and a range of variability of $\pm 2.12 \mathrm{mmHg}$, both increased with respect to the results found with the constant capacitance. 


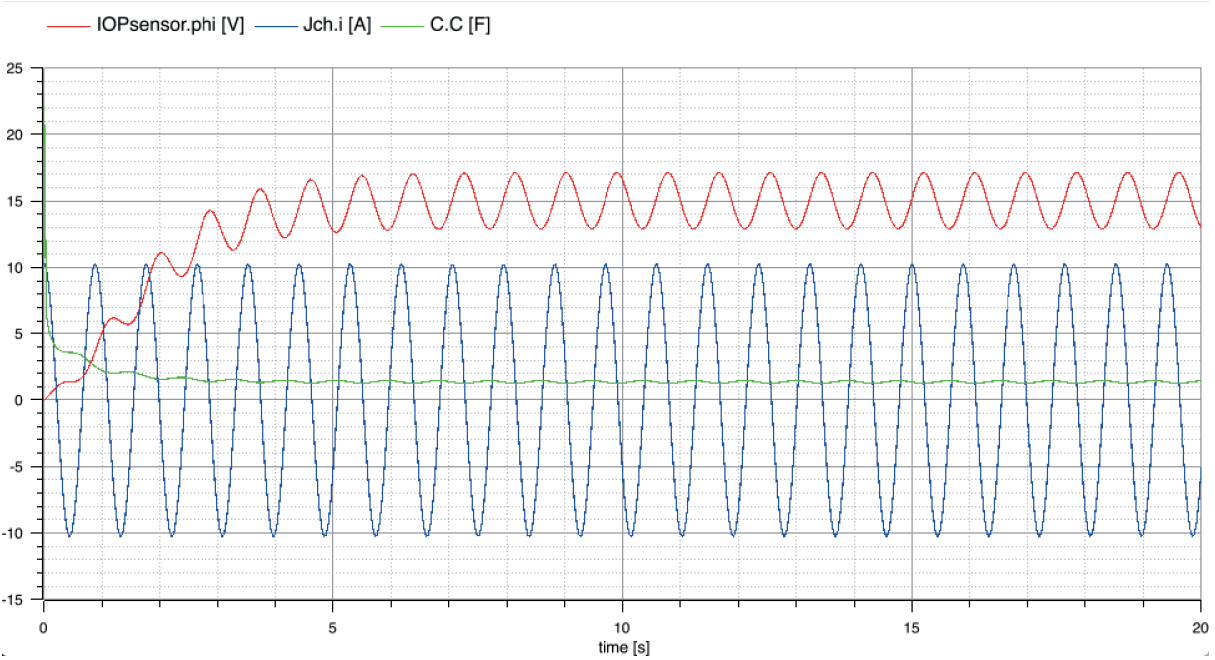

Fig. 3. OpenModelica simulation output: IOP, current generator Jch, and capacitor C.

\section{Conclusions}

This project provides a first attempt to consistently combine the static and dynamic components contributing to the pressure distribution inside the eye. It will help elucidate the relationship between the measured values of IOP and some important factors that may vary among patients. In a long-term perspective, the software developed within this project will be integrated within a larger virtual simulator for ocular biophysics, which may lead to a new tool for clinical use aimed at providing physicians with an integrated view of the patient's status to monitor, prevent, and treat diseases in a patient-specific manner.

\section{References}

1. Open Source Modelica Consortium (OSMC), Linköping, Sweden.

2. Szopos M, Cassani S, Guidoboni G, Prud'homme C, Sacco R, Siesky B, Harris A. Mathematical modelling of aqueous humor flow and intraocular pressure under uncertainty: towards individualized glaucoma management. Journal for Modeling in Opthalmology. 2016;1(2):29-39.

3. Kiel JW, Hollingsworth M, Rao R, Chen M, Reitsamer HA. Ciliary blood flow and aqueous humor production. Prog Retin Eye Res. 2011;30(1):1-17.

4. Krakau CE. Calculation of the pulsatile ocular blood flow. Invest Ophthalmol Vis Sci. 1992;33(9):27542756.

5. Friedenwald JS. Contribution to the theory and practice of tonometry. Am J Ophthalmol. 1937;20(10):985-1024. 